\title{
Study of Comprehensive Traffic Planning In Europe
}

\author{
Huanyu Yang \\ China Academy of Transportation Science, Beijing 100029, China \\ yanghuanyuzhou@126.com
}

Keywords: traffic planning, transportation policy, evaluation index system.

\begin{abstract}
This passage, through field study, elaborates comprehensive traffic planning in Germany, Europe, from the levels of comprehensive traffic planning, the methods and targets of comprehensive traffic planning, the evaluation index system of comprehensive traffic planning and the public participation of the planning. It provides reference to the comprehensive traffic planning in our country.
\end{abstract}

\section{The levels of comprehensive traffic planning in Germany}

German traffic development planning is divided into three sections: the traffic development planning, the spatial development planning, a coordinated planning between various departments.

There are several levels of traffic development planning: the top development planning is the pan European transport planning, providing guidelines for the entire European traffic planning; next one is the German Federal road traffic planning, defining which railway and highway construction is on the national level; the next one is traffic development planning in every state; regional traffic planning;City public traffic planning; and the last one is local transportation planning.

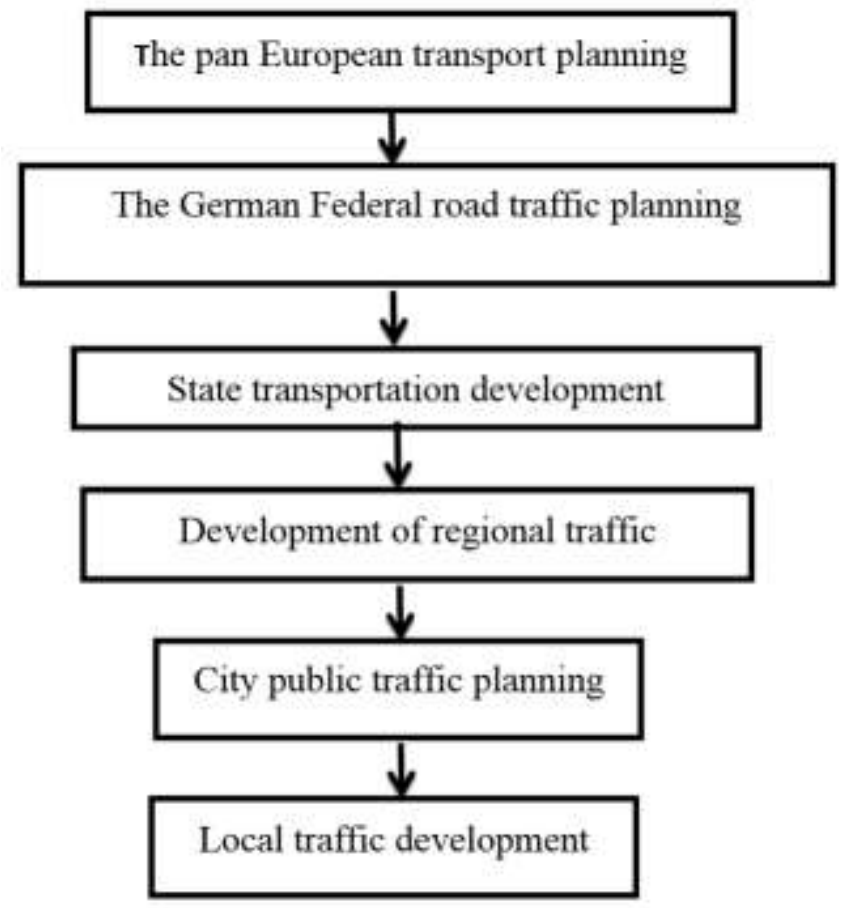

Fig. 1 Schematic diagram of the hierarchy of German traffic development planning

Then introduce the spatial development planning. The hierarchical structure of the transportation development planning and spatial development planning is similar. 


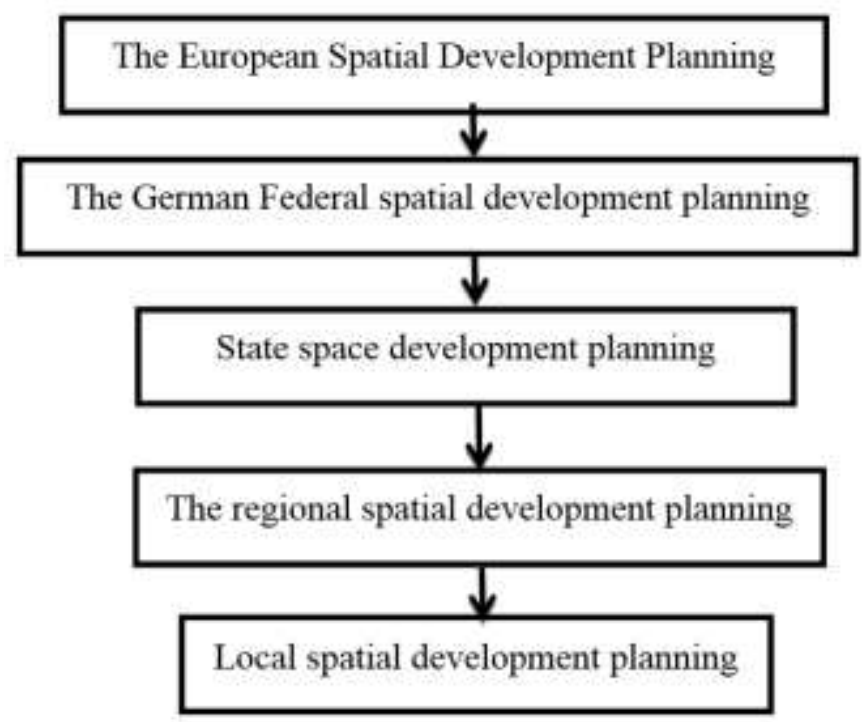

Fig. 2 Schematic diagram of the hierarchy of German spatial development planning

The last is the coordinated planning between various departments. Traffic planning involves many departments, such as the economy, education, culture, climate etc. There should be a good communication among relevant departments in the planning. And there should be a link between the relevant content and the interdepartment planning.

\section{The methods, targets and problems of comprehensive traffic planning in Germany}

The German Federal Ministry of transport is currently developing a new traffic planning, expected to be completed in 2014. Now the planning in use was made in 2008, including highway, railway, air cleaning, noise reduction, relating to many aspects of infrastructure, management and information etc.

The goals of transportation development planning must be sustainable, thus, in planning, not only the traffic infrastructure should be considered, but also the operating, management, cost should be considered.The transportation development planning primarily include 3 aspects: traffic, environment and society.

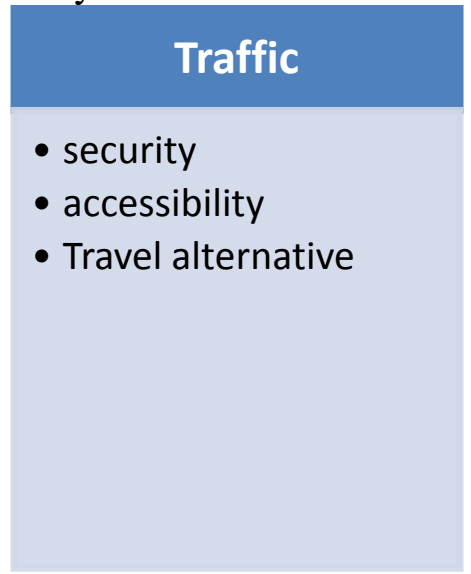

Environment
- clean air
- noise reduction
- natural landscape
protection
- enhancing the quality
of city construction
- energy efficiency
increase

\section{Society}

- affordability

- payablility

- friendly to children

- barrier free for the elderly and the disabled

- reasonable allocation

- reduction of isolation

Fig. 3 Goals of transportation development planning

The longer the periods of traffic planning, the bigger the deviation is from planning goals to the realization. Thus, local traffic development planning should be typically reenacted 5-10 years, and the effect should be evaluated and supervised. If there is deviation in the goal of planning, the plan should be revised, new measure should be used.

The existence problems and challenges of the current traffic development are mainly five aspects: the first one is that the population is changing. The second one is that the climate is changing. The third one is the financial problems, in the future the transportation infrastructure construction and 
maintenance funding gap will continue to expand. The fourth is environmental issue. And the last one is the problem of relationship between city sustainable development and traffic.

\section{The evaluation index system of comprehensive traffic planning in Germany}

The evaluation index system of the transportation development planning includes planning index, traffic index, safety index, and environmental index.

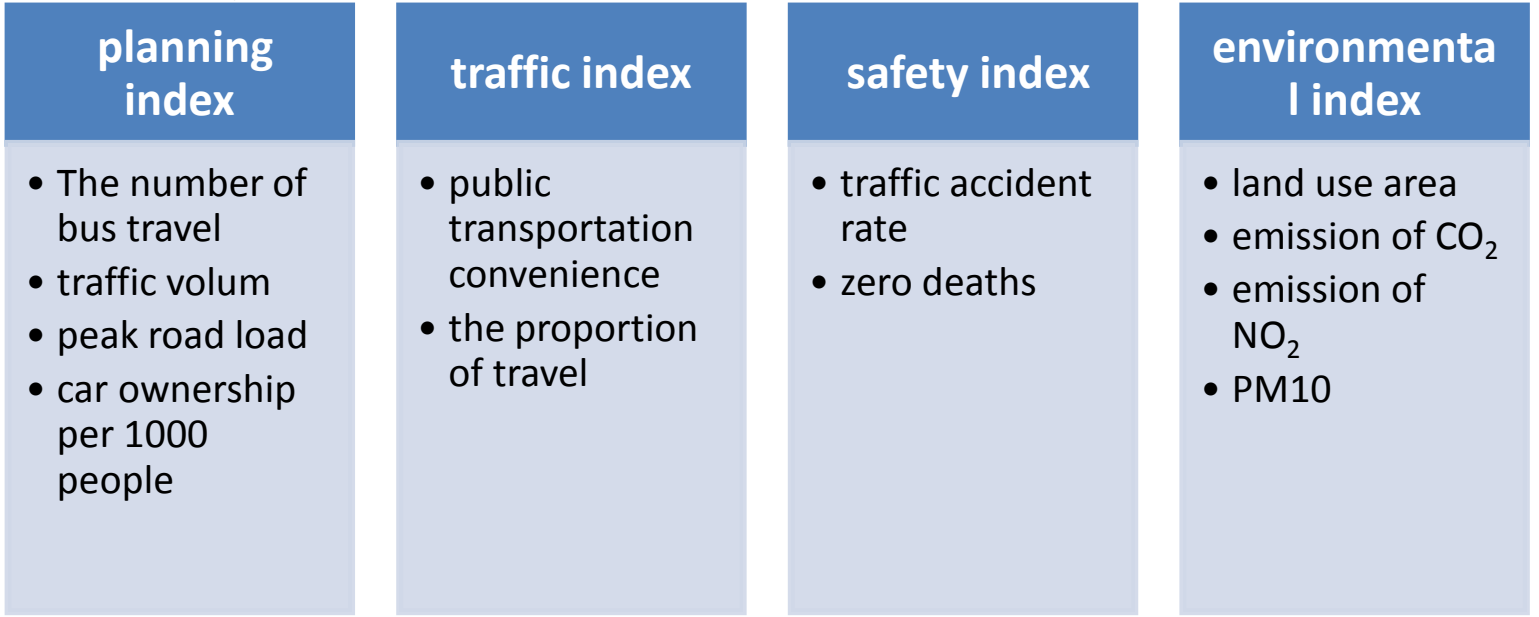

Fig. 4 The evaluation index system of the transportation development planning

\section{The public participation of the comprehensive traffic planning in Germany}

The public participation of German traffic planning began in 2011, resulting from the building of No. 21 train station in Stuttgart. Because the construction of the station caused a large-scale conflict between the public and the police, a widespread concern was caused, then public participation has been added to traffic planning. Formulation of German traffic development planning needs the consent of the government departments' agreement, traffic development planning should be evaluated by experts, and public participation in planning is required, and only after approval by the city authorities can the planning be taken effect.

\section{The enlightenment to China}

5.1 Improve the comprehensive transportation planning mechanism and secure level planning.

Deepening the reform of the management system of each department and promoting the reform of "big department system" provide the safeguard to the transport planning. Further rationalize the relations of each department in the planning, to establish a clear hierarchy, orderly collaboration architecture. Integrate railway, highway, water transport, aviation, telecommunications and city traffic planning, providing the public fast, safe, comfortable, convenient integrated transport service.

\subsection{Make relevant policies and regulations standards.}

Establish the legal status of the comprehensive planning, enhance the authority of the traffic planning system; establish the hierarchical traffic planning system, clear planning body, coordinate the relationship between the relevant planning; establish the planning procedure covering planning evaluation, approval, implementation, revision procedures and authority etc. Implement the system of examination and evaluation indexes of comprehensive transportation planning, ensure the smooth implementation of index.

\section{References}

[1]. Hongzhen Zhao. Enlightenment of EU transportation policy to China. 《China Investment》. 2013(S1):101-101.

[2]. Information on: https://ec.europa.eu/. 
[3]. Zhaoran Liu. Enlightenment of European Union's multimodal transport policy on the development of railway-river combined transportation in China. 《Railway Economics Research》. 2013, 35(5):56-60.

[4]. Xinjun Zhou. EU Low-carbon Transport Strategic Initiatives and Its Implications. 《SINOGLOBAL ENERGY》. 2012, 17(11):6-14

[5]. Min Huang. Development Strategy and Enlightenment of Foreign Transportation. 《Railway Economics Research》. 2007(2):28-28 